\title{
Preparation and Characterization of Polysulfone Based Hollow Fibre Composite Membranes for Water Purification
}

\author{
A. M. Vijesh ${ }^{\mathrm{a}^{*}}$ P. C. Shyma ${ }^{\mathrm{b}}$, V. Prakash ${ }^{\mathrm{c}} \&$ B. Garudachari ${ }^{\mathrm{d}}$ \\ ${ }^{a}$ Department of Chemistry, Payyanur College, Payyanur, Kannur, \\ 670327 Kerala, India

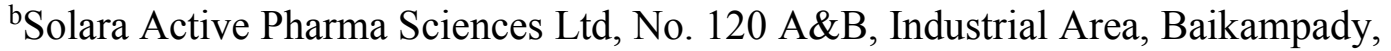 \\ 575014 Mangalore, India \\ ${ }^{\mathrm{c}}$ Department of Physics, Payyanur College, Payyanur, Kannur, 670327 Kerala, India \\ ${ }^{\mathrm{d}}$ Membrane Technology Laboratory, Chemistry Department, NIT-Karnataka, \\ Surathkal, Mangalore 575 025, India
}

Submitted: 21/6/2018. Revised edition: 8/8/2018. Accepted: 11/8/2018. Available online: 21/11/2018

\begin{abstract}
Nanofiltration membranes are gaining more importance in the field of water treatment especially in desalination plants. Hollow fibre membranes have been preferred over other membrane configurations due to their high membrane surface area to module volume, mechanical property and easy handling. In the present work, we prepared new type of polysulfone (PSf) composite hollow fibre membranes by blending PSf with polyvinylpyrrolidinone-nitrobenzene (PVPD) in different compositions. New membranes were fabricated using wet-jet phase inversion technique. The resultant composite membranes were characterized by various analytical techniques such as water contact angle, SEM, DSC, TG. Pure water flux of the membranes was measured using cross-flow filtration techniques. The study revealed that increased composition of PVPD in casting solution resulted in a highly porous membrane structure and the pure water flux of the membranes increases in the same order.
\end{abstract}

Keywords: PVP, PSf, hollow fibre membrane, composite, desalination, nanofiltration

\subsection{INTRODUCTION}

Membrane filtration has gained enormous applications in the field of separation technology [1]. It is useful in various industrial fields such as pharmaceutical, biomedical, food, pure water production, desalination and purification of gases [2-5]. The pore size, surface roughness, mechanical strength and hydrophilicity of the membrane are the various factors influences the membrane separation process [6]. Polysulfone (PSf) is a commonly used membrane material due to its excellent mechanical, chemical and thermal properties $[4,7$,
8]. It can resist extreme $\mathrm{pH}$ conditions of the medium and also having good film forming capacity [6]. However, the highly hydrophobic nature of the PSf membranes limits its application as a membrane material.

The membrane fouling due to the hydrophobicity of the polymeric membrane is also a major challenge for the researchers. There are several studies reported in the literature to make the PSf membranes more hydrophilic, such as coating, grafting and blending [9]. But blending with a hydrophilic polymer is the best method due to its simplicity [6]. Blending of PSf with hydrophilic polymers can change the 
nature of the surface and the pore size of the membrane and thereby improving the membrane performance with high permeability and antifouling property [10].

There are two types of membranes used by industries, flat sheet and hollow fibre. Hollow fibre membranes are having high permeability due to its large surface area. Cross-flow method can be easily adopted in the case of hollow fibre membranes and hence fouling of the membrane surface will be very less as compared to a flat sheet membranes [11]. Diverse methodologies have been developed by many researchers to enhance its hydrophilic character such as, chemical surface treatment and the addition of hydrophilic additives $[12,13,14]$. From the literature, it was clear that addition of the hydrophilic organic polymer polyvinylpyrrolidinone into the casting solution is capable to improve the hydrophilicity, porosity, permeability and antifouling nature of the resultant membrane [13].

In the present work new polymeric PSf-PVPD (polyvinylpyrrolidinonenitrobenzene) hollow fibre membranes have been prepared by wet-jet phase inversion technique. The proton donor (-NH-) and proton acceptor $(-\mathrm{N}=)$ hydrogen bonding sites in PVPD forms miscible blend with PSf [6]. We hope that the hydrophilic nature of the PVPD will improve the water flux of the composite membranes when compared with the hydrophobic PSf membrane. We were also expected that the addition of PVPD would alter the surface morphology of the membranes and thereby exhibit good salt rejection properties. The results of such studies have been discussed in terms of the composition of the blend membranes.

The characterizations of the membranes were done by performing its water contact angle, scanning electron microscopy (SEM), differential scanning calorimetry (DSC), thermogravimetry (TGA). The permeability of the membranes was studied by conducting pure water flux experiments in a cross-flow mode. Results of such studies were discussed in this paper.

\subsection{METHODS}

\subsection{Materials}

Polysulfone (PSf, MW=22,000 Da), 1methyl-2-pyrrolidone (NMP) and Polyvinylpyrrolidone (PVP) from Sigma-Aldrich Co. (USA) were used in the casting solution as the base polymer, the solvent and the pore former respectively. Commercially available hydrazine hydrate and 2-nitro benzaldehyde were procured from Sigma Aldrich.

Morphologies of the prepared membranes were examined by SEM (Carl Zeiss Ultra Plus). The water contact angles for the membranes were measured at room temperature using sessile droplet method by a KSV Attension contact angle analyser. Exstar DSC 7020 differential scanning calorimeter was used to investigate the miscibility of polymers. The TG curves were obtained by using a Seiko Exstar 7200 thermal analyser under air atmosphere.

\subsection{Synthesis of Polyvinylpyrrolidone- none-nitrobenzene Polymer (PVPD)}

\subsection{1 \\ Synthesis \\ Polyvinylpyrrolidone Hydrazide}

of

Mixture of polyvinylpyrrolidone $(5 \mathrm{~g})$, hydrazine hydrate $(10 \mathrm{~mL})$ and ethanol $(50 \mathrm{~mL})$ were stirred at $75^{\circ} \mathrm{C}$ for $48 \mathrm{hrs}$ under nitrogen in a round bottom flask. After completion of the reaction, the reaction mixture was concentrated under vacuum and creamy solid material was washed with a mixture of 
diethyl ether and hexane. The colourless hygroscopic solid compound was stored in nitrogen atmosphere. The yield of the hydrazide is $3.2 \mathrm{~g}$.

\subsubsection{Synthesis \\ Polyvinylpyrrolidone (PVPD)}

Polyvinylpyrrolidone hydrazide $(2.5 \mathrm{~g})$ was dissolved in $25 \mathrm{~mL}$ of ethanol. 2Nitrobenzaldehyde $(5 \mathrm{~g})$ and acetic acid $(0.5 \mathrm{~mL})$ was added to the above solution and stirred at $60^{\circ} \mathrm{C}$ for $48 \mathrm{hrs}$. It was then quenched in ice cold water, the product was filtered and washed with water, followed by ethanol. The yellow product was then dried in an oven at $60^{\circ} \mathrm{C}$ for $24 \mathrm{hrs}$. Figure 1 depicts the IR and NMR spectra of PVPD and both the spectra were matching with the actual structure of the polymer. The yield of PVPD obtained was $1.8 \mathrm{~g}$.

\subsection{Synthesis of PSf Hollow Fibre Membranes}

Polyvinylpyrollidinone, PVP (3g), as pore former, was dissolved in $79 \mathrm{~mL}$ of

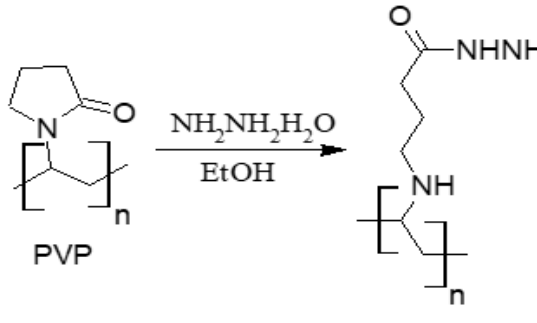

NMP by constant stirring and heating at $120^{\circ} \mathrm{C}$. PSf $(18 \mathrm{~g})$ was then added to the PVP-NMP solution and the mixture was stirred at $100^{\circ} \mathrm{C}$ for 12 hours to obtain a homogeneous solution. The resulting solution was cooled, and sonicated for 30 minutes to remove any air bubbles. The resultant viscous PSf/PVP/NMP solution was extruded through a spinneret to form hollow fibre membrane. A tube-in-orifice spinneret was used for the spinning of fibres (see Figure 2). The spinning conditions that applied to fabricate the hollow fibre membrane are given in Table 1.

Table 1 Spinning parameters for the membrane fabrication

\begin{tabular}{ll}
\hline Parameters & Value \\
\hline Dope extrusion rate & $1 \mathrm{~mL} / \mathrm{min}$ \\
Bore fluid & Distilled water \\
Bore fluid rate & $1 \mathrm{~mL} / \mathrm{min}$ \\
Collection drum rate & $10 \mathrm{~m} / \mathrm{min}$ \\
Air gap distance & $1 \mathrm{~cm}$ \\
Spinneret outer & $0.6 \mathrm{~mm} /$ \\
\hline
\end{tabular}

After spinning, the membrane was immersed in a coagulation bath

Scheme 1 Synthetic route for the preparation of PVPD polymer

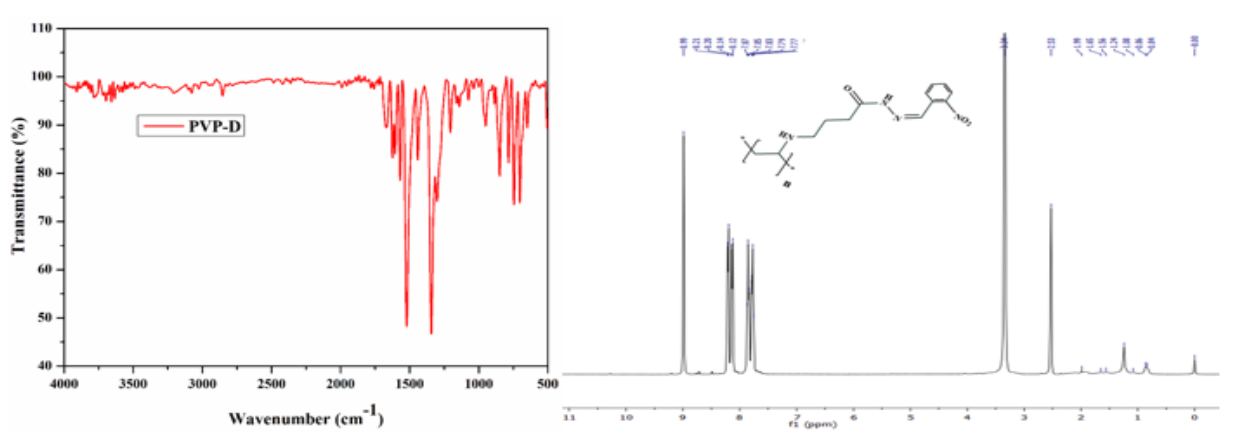

Figure 1 IR and ${ }^{1} \mathrm{H}$ NMR spectra of the prepared PVPD polymer 


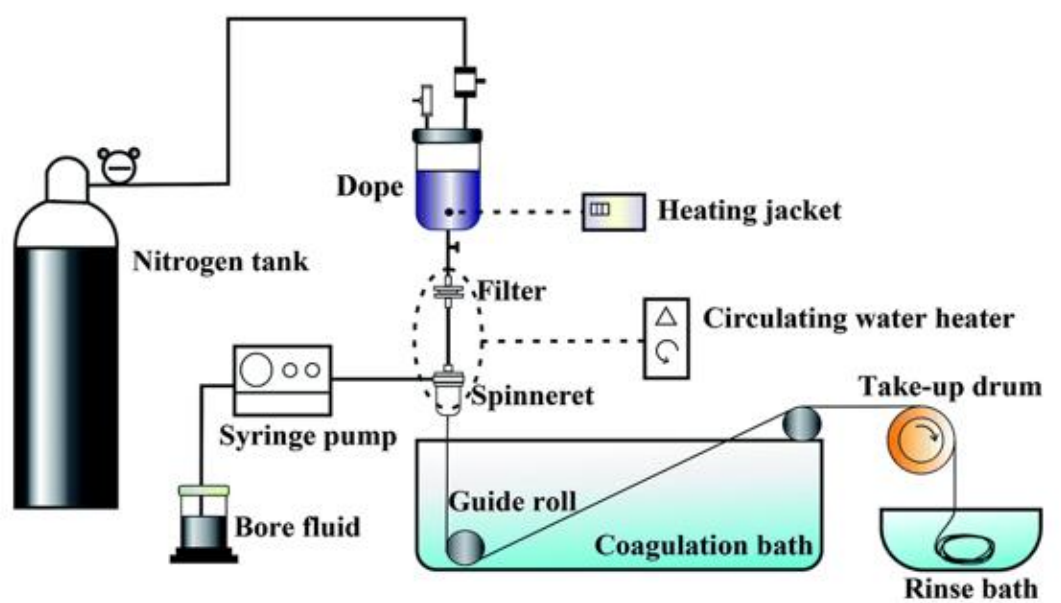

Figure 2 A schematic representation and machine of hollow fiber fabrication [17]

containing distilled water for 24 hours to remove the residual solvent. It was then treated with ethanol:water mixture (50:50 wt\%) for 1 hour followed by in pure ethanol for another 1 hour for improving the membrane wettability and preventing pore collapse. The membrane was then air dried by hanging vertically at room temperature for 3 days $[15,16]$.

\subsection{Synthesis of PSf-PVPD Hollow Fibre Blend Membranes}

PVP as pore former $(3 \mathrm{~g})$ was dissolved in $79 \mathrm{~mL}$ of NMP by constant stirring and heating at $120^{\circ} \mathrm{C}$. Required quantity of PVPD was then dissolved in PVPNMP solution at $120^{\circ} \mathrm{C}$ and the mixture was kept under stirring at $100^{\circ} \mathrm{C}$ for 2 hours to obtain a clear solution. PSf (18g) was then added to the PVPDPVP-NMP solution and the mixture was stirred at $100^{\circ} \mathrm{C}$ for 12 hours to get a homogeneous solution.

The resulting solution was cooled, and degassed in an ultrasonic bath to remove any bubbles. The resultant viscous solution was extruded through a spinneret to form hollow fibre membranes. The membranes were immersed in a coagulation bath containing distilled water for $24 \mathrm{hrs}$ to remove the residual solvent. It was then treated with ethanol:water mixture (50:50 wt $\%$ ) for 1 hour followed by pure ethanol for another 1 hour. Membranes were then air dried for 3 days. Characterization of the prepared membranes is given in Table 2 and the photo of each dope is shown in Figure 3.

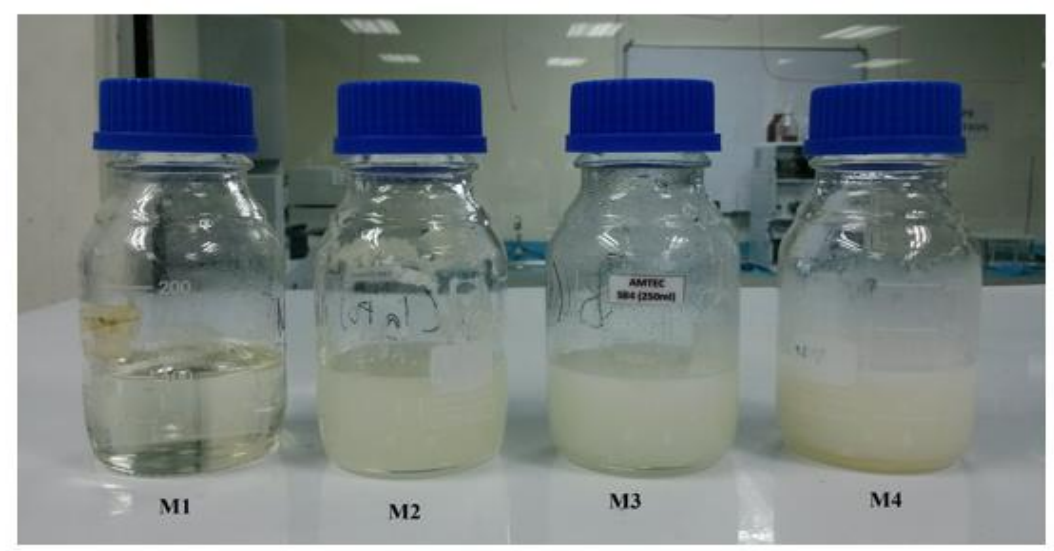

Figure 3 Images of viscous liquids for the fabrication of M1, M2, M3 and M4 
Table 2 Characterization of prepared membranes

\begin{tabular}{ccccc}
\hline Membr & $\begin{array}{c}\text { PSf } \\
(\mathbf{g})\end{array}$ & $\begin{array}{c}\text { PVPD } \\
(\mathbf{g})\end{array}$ & $\begin{array}{c}\text { PVP } \\
(\mathbf{g})\end{array}$ & $\begin{array}{c}\text { NMP } \\
(\mathbf{m L})\end{array}$ \\
\hline M1 & 18 & 0 & 3 & 79 \\
M2 & 18 & 1 & 3 & 79 \\
M3 & 18 & 3 & 3 & 79 \\
M4 & 18 & 5 & 3 & 79 \\
\hline
\end{tabular}

\subsection{Characterization of PSf-PVPD Hollow Fibre Blend Membranes}

The morphology of the prepared membranes was examined by using Scanning Electron Microscopy (SEM) analysis. Thermal stability of the membranes was illustrated by the DSC and TGA studies.

The wettability of the membranes was studied by measuring the contact angle of water for the membranes at room temperature. Five measurements were made for each sample and then the average value was reported. The pure water flux was calculated by performing filtration experiments in a cross-flow mode.

\subsection{Filtration Performance of the Membranes}

Pure water permeability was calculated by measuring the pure water flux as given in [18]. A bunch of 10 hollow fibre membranes with an approximate length of $23.5 \mathrm{~cm}$ was potted into a steel tube using epoxy resin. The module was then fitted into a filtration set up. Filtration experiment was performed in a cross-flow mode. All the prepared membranes were tested and their flux was measured. Compressed distilled water was used as permeate for the filtration measurements. All the experiments were performed at room temperature and the membranes for testing were soaked in distilled water for one day before conducting the studies.
The pure water flux measured at 0.1 $\mathrm{MPa}$ was calculated as follows:

$F=\frac{V}{A t}$

Where $\mathrm{F}$ is the flux $\left(\mathrm{Lm}^{-2} \mathrm{~h}^{-1}\right)$ and $\mathrm{V}$ is the volume of permeate (L) at time $t(h)$. $\mathrm{A}$ is the effective filtration area of the membrane $\left(\mathrm{m}^{2}\right)$. Before taking the readings, permeate was allowed to attain equilibrium flow for at least 15 $\min$. The results are tabulated in Figure 5.

\subsection{RESULTS AND DISCUSSION}

\subsection{Scanning Electron Microscopy (SEM)}

Figure 4 presents the cross-sectional SEM images of the fabricated membranes M1, M2, M3 and M4. It was observed from the SEM images that the overall cross-section of hollow fibres are not perfectly cylindrical especially the bore. This could jeopardize the mechanical integrity of the membranes under high operating pressures. From SEM images it was clear that the finger-like structure becomes more apparent with the addition of PVPD into the spinning dope. This is due to high hydrophilicity nature of PVPD which enhances the phase inversion during the spinning process. The finger-like structure is narrower with increasing PVPD and the skin layer becomes thinner. We can expect a higher water permeation flux from such morphology.

\subsection{Contact Angle of the Membranes}

Contact angle measurement shows that there is no much variation in the angle with the addition of PVPD. Moreover, there is a slight increase in the contact angle with the increase in the percentage of PVPD in the membranes. 

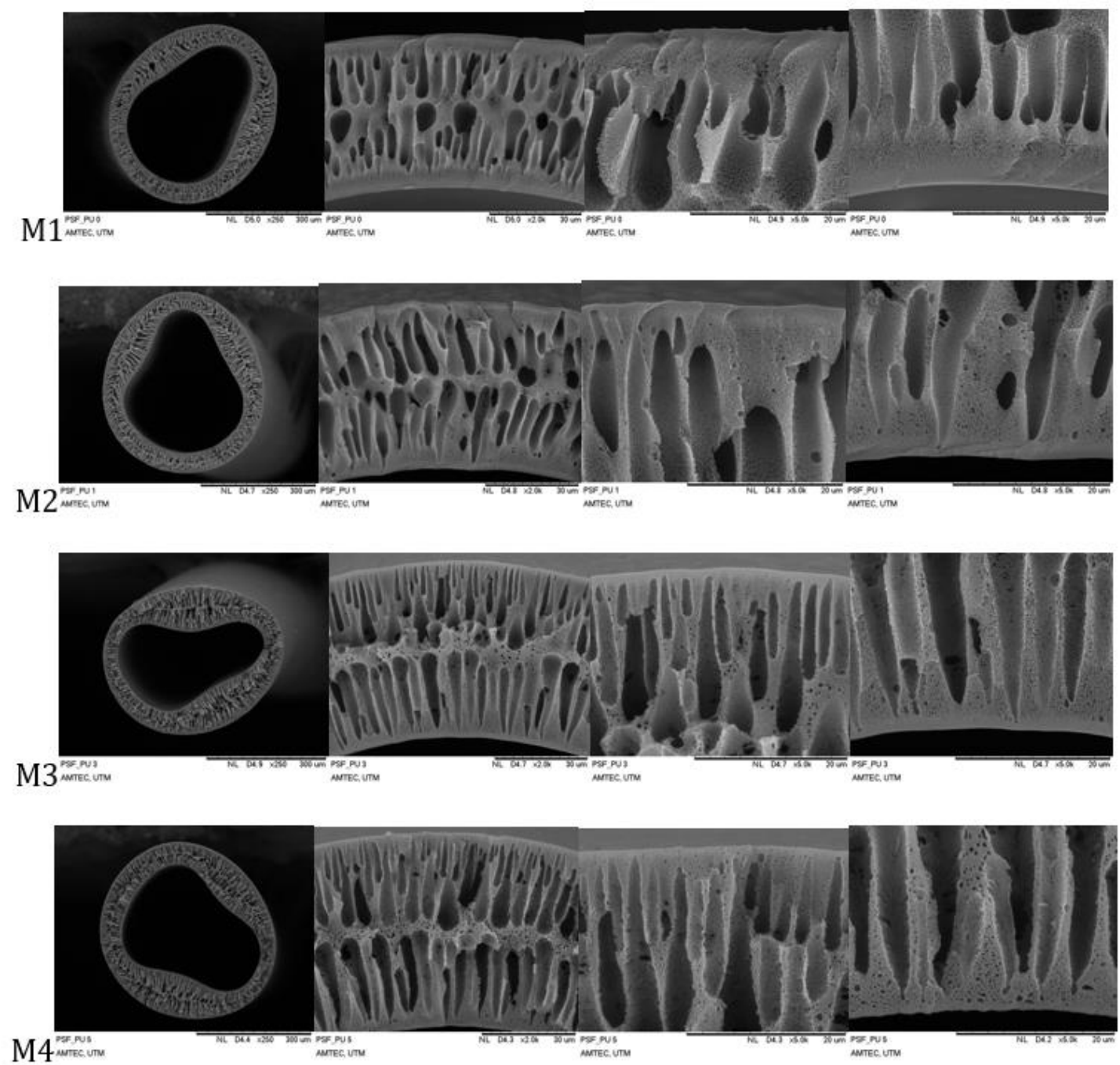

Figure 4 Cross-sectional SEM images of the membranes M1, M2, M3 and M4

Contact angles of the prepared membranes M1, M2, M3 and M4 are given in Table 3 and graphically represented in Figure 6.

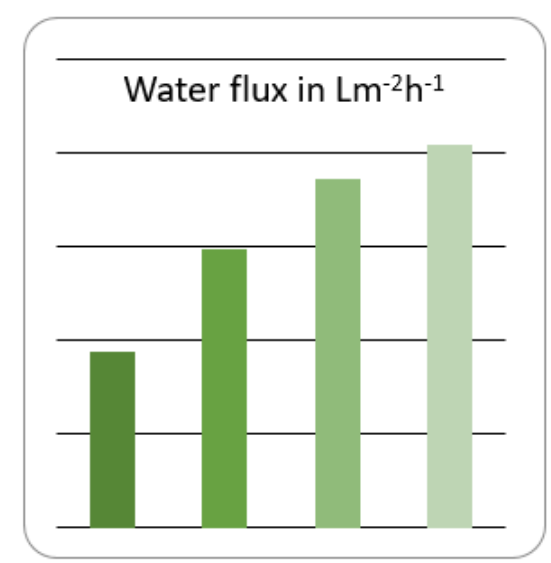

Figure 5 The pure water flux of the membranes at $0.4 \mathrm{MPa}$ pressure
Table 3 Contact angles of fabricated membranes

\begin{tabular}{cc}
\hline Membranes & Contact angle \\
\hline M1 & $87.80 \pm 3.50$ \\
M2 & $87.03 \pm 1.67$ \\
M3 & $87.77 \pm 1.69$ \\
M4 & $89.44 \pm 3.34$ \\
\hline
\end{tabular}

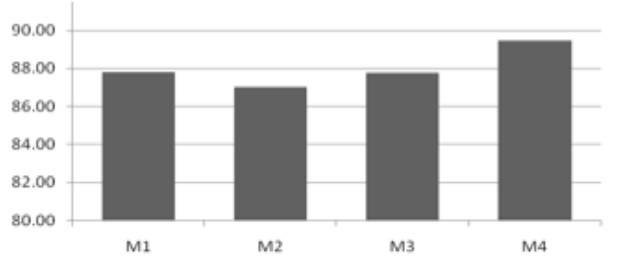

Figure 6 Contact angle of the membranes M1, M2, M3 and M4 


\subsection{DSC and TGA Studies}

The thermal stability of the membrane produced in this study was analyzed by thermal gravimetric analyser (TGA) to measure weight loss as a function of temperature. The experiments were performed with a TGA apparatus (TGA 2950 Dupont Instruments) from 30 to $800^{\circ} \mathrm{C}$ at $10^{\circ} \mathrm{C} \mathrm{min}^{-1}$ under nitrogen atmosphere, with a nitrogen flow rate of $20 \mathrm{~mL} \mathrm{~min}^{-1}$.

TGA curves for the hollow fibres fabricated are demonstrated in Figure 7. There is no clear trend in thermal stability could be seen from the curves.

The glass transition temperature $\left(T_{g}\right)$ of the hollow fibre membranes were measured using differential scanning calorimetry (Mettler Toledo DSC 822e). The membrane sample was cut into small pieces, weighed and placed into a pre-weighed aluminium crucible. Each membrane specimens was heated at temperature ranges of 15 to $300{ }^{\circ} \mathrm{C}$ at a heating rate of $10{ }^{\circ} \mathrm{C} \mathrm{min}{ }^{-}$ ${ }^{1}$ then was cooled back to $15^{\circ} \mathrm{C}$ with cooling rate of $10{ }^{\circ} \mathrm{C} \mathrm{min}^{-1}$. The

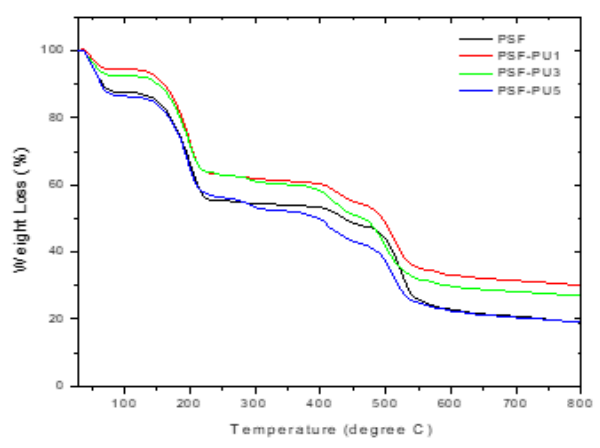

Figure 7 TGA curves of the membranes $\mathrm{M} 1, \mathrm{M} 2, \mathrm{M} 3$ and M4

specimen was heated back to $300{ }^{\circ} \mathrm{C}$ at the same rate. The first heating and cooling cycle aim to remove the thermal history of the sample. $T_{g}$ of the sample was determined from the midpoint temperature of the transition region in the second heating cycle. DSC thermogram of the membranes is shown in Figure 8.

DSC results for the hollow fibres are exemplified in Table 4. From the table, it is clear that the glass transition temperatures of the blend membranes are decreasing significantly with an increasing portion of PVPD in the spinning dope.

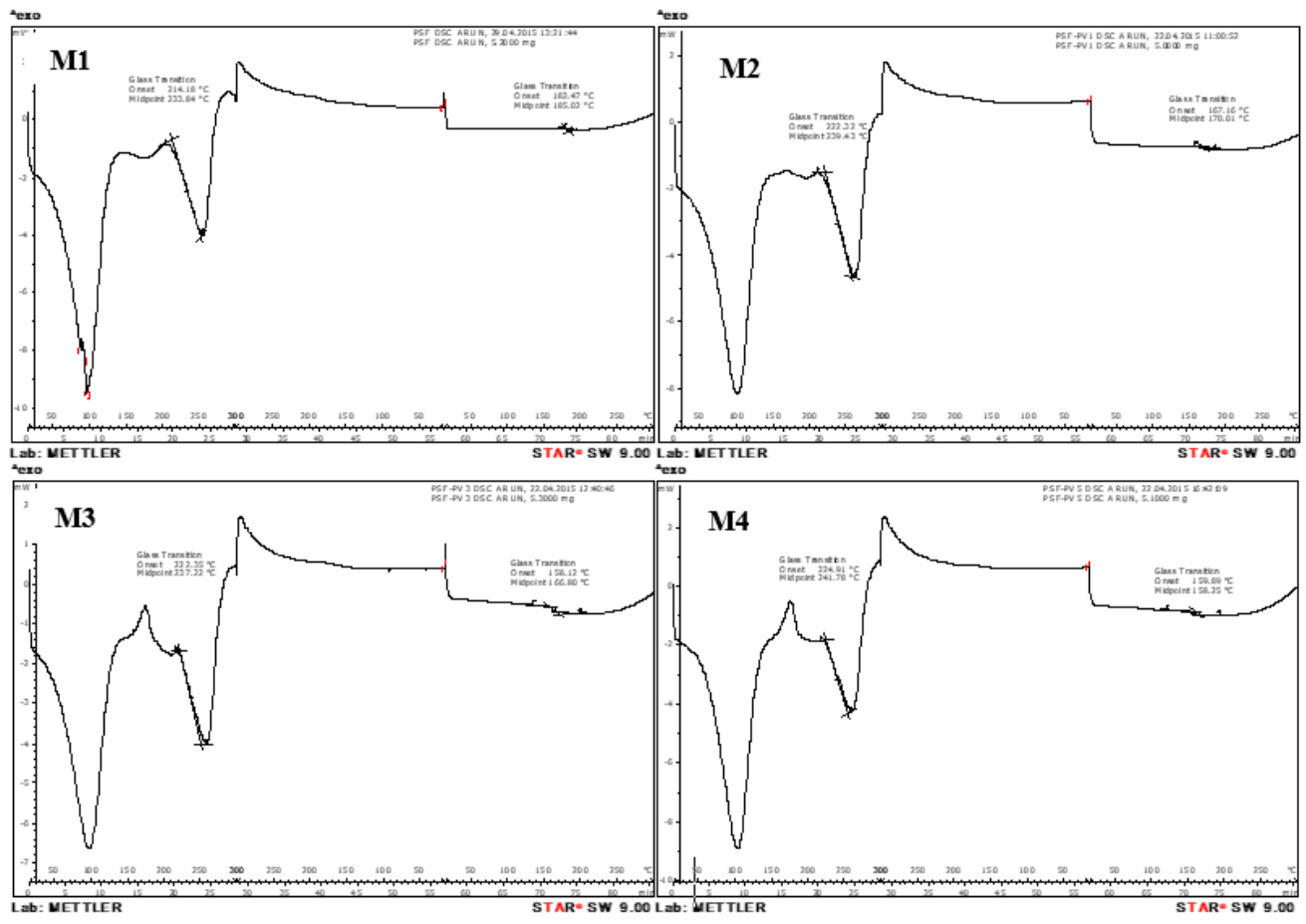

Figure 8 Glass transition temperature of the membranes M1, M2, M3 and M4 
Table 4 Glass Transition Temperature

\begin{tabular}{cc}
\hline Membranes & $\mathbf{T g}\left({ }^{\circ} \mathbf{C}\right)$ \\
\hline M1 & 185.02 \\
M2 & 170.01 \\
M3 & 166.80 \\
M4 & 158.25 \\
\hline
\end{tabular}

\subsection{CONCLUSION}

In summary, PSf-PVPD hollow fibre blend membranes were synthesized by phase inversion method via the reaction between polysulfone, PVPD and PVP (as pore former) in $\mathrm{NMP}$ at $100^{\circ} \mathrm{C}$ under constant stirring and heating almost for 24 hours.

Different compositions of reactants were used for the membrane fabrication. The characteristic study of fabricated membranes was done by examining the SEM images and the DSC analysis of each. The increased composition of PVPD in casting solution resulted in a highly porous membrane structure.

Highly porous and hydrophilic nature of PSf-PVPD membranes contributed to the enhanced flux. Contact angle measurements showed not much difference in the angle with the increase in PVPD content. Rather there is a slight increase in the contact angle shows more hydrophobic nature of the membranes. But the increase in pure water flux can be explained by the hydrophilic nature of the PVPD and also by the highly porous structure of the composite membranes.

Further, the fabricated membranes can be tested for their rejection properties. Hope these hollow fibre membranes will be a better choice for the water purification especially for the removal of salts, heavy metals and dyes from polluted water.

\section{ACKNOWLEDGEMENT}

AMV thanks to Dr. Arun M. Isloor, NITK, Surathkal, India for his constant support and technical help. The authors would like to thank AMTEC, UTM, Malaysia for giving permission to use their lab facilities for fabricating the hollow fibre membranes and their help in recording the SEM, DSC, contact angle of the composite membranes. Authors are also thankful to Principal and management of Payyanur College for providing facilities to finish this work.

\section{REFERENCES}

[1] A. M. Vijesh, J. Liya, P. C. Shyma. 2017. ZnO Nano Composite Membranes for Desalination. J. Applied Membrane Science \& Technology. 21: 43-48.

[2] V. R. Pereira, A. M. Isloor, A. K. Zulhairun, W. J. Lau, A. F. Ismail. 2016. Preparation of Polysulfone based PANI-TiO2 Nanocomposite Hollow Fiber Membranes for Industrial Dye Rejection Applications. RSC Adv. 6: 99764-99773.

[3] S. S. Shenvi, A. M. Isloor, A. F. Ismail. 2015. A Review on RO Membrane Technology: Developments and Challenges. Desalination. 368: 10-26.

[4] N. A. A. Hamid, A. F. Ismail, T. Matsuura, A. W. Zularisam, W. J. Lau, E. Yuliwati, M. S. Abdullah. 2011. Morphological and Separation Performance Study of Polysulfone/titanium Dioxide $\left(\mathrm{PSF} / \mathrm{TiO}_{2}\right) \quad$ Ultrafiltration Membranes for Humic Acid Removal. Desalination. 273: 8592.

[5] X. Li, S.D. Feyter, I. F. J. Vankelecom. 2008. 
Poly(sulfone)/sulfonated Poly (ether ether ketone) Blend Membranes: Morphology Study And Application in the Filtration of Alcohol Based Feeds. $J$. Membr. Sci. 324: 67-75.

[6] E. Erenn, A. Sarihan, B. Eren, H. Gumus, F. O. Kocak. 2015. Preparation, Characterization and Performance Enhancement of Polysulfone Ultrafiltration Membrane using PBI as Hydrophilic Modifier. J. Membr. Sci. 475: 1-8.

[7] J. Miao, G. Chen, C. Gao, C. Lin, D. Wang, M. Sun. 2006. Preparation and Characterization of N,O Carboxy Methylchitosan (NOCC)/ Polysulfone (PS) Composite Nano Filtration Membranes. J. Membr. Sci. 280: 478-484.

[8] K. B. Prabhu, M. B. Saidutta, A. M. Isloor, R. Hebbar. 2017. Improvement in Performance of Polysulfone Membranes through the Incorporation of Chitosan-(3phenyl-1h-pyrazole-4-

carbaldehyde. Cogent

Engineering. 4: 1403005.

[9] V. R. Pereira, A. M. Isloor, U. K. Bhat, A. F. Ismail, A. Obaid, H. K. Fun. 2015. Preparation and Performance Studies of Polysulfone-sulfated Nano-titania $\left(\mathrm{S}-\mathrm{TiO}_{2}\right)$ nanofiltration Membranes for Dye Removal. RSC Adv. 5: 53874-53885.

[10] Y. L. Liu, C. H. Yu, L. C. Maa, G. C. Lin, H. A. Tsai, J. Y. Lai. 2008. The Effects of Surface Modifications on Preparation and Pervaporation Dehydration Performance of Chitosan/ Polysulfone Composite HollowFiber Membranes. J. Membr. Sci. 311: 243-250.

[11] H. A. Tsai, Y. S. Ciou, C. C. Hu, K. R. Lee, D. G. Yu, J. Y. Lai. 2005. Heat-treatment Effect on the Morphology and Pervaporation Performances of Asymmetric PAN Hollow Fiber Membranes. J. Membr. Sci. 255: 33-47.

[12] P. Kanagaraj, N. Alagumalai, D. Rana, T. Matsuura, S. Neelakandan, K. Malarvizhi. 2015. Effects of Poly (Vinyl Pyrrolidone) on the Permeation and Fouling-Resistance Properties of Poly (Ether Imide) Ultra-Filtration Membranes. Ind. Eng. Chem. Res. 54: 4832-4838.

[13] P. Wang, J. Ma, Z. Wang, F. Shi, Q. Liu. 2012. Enhanced Separation Performance of PVDF/PVP-g-MMT

Nanocomposite Ultrafiltration Membrane based on the NVPgrafted Polymerization Modification of Montmorillonite (MMT). Langmuir. 28: 47764786.

[14] J. Pieracci, J. V. Crivello, G. Belfort. 2002. UV-assisted Graft Polymerization of N-vinyl-2Pyrrolidinone onto Poly (ether sulfone) Ultrafiltration Membranes Using Selective UV Wavelengths. Chem. Mater. 14: 256-265.

[15] G. Bakeri, A. F. Ismail, M. R. D. Arzhandi, T. Matsuura. 2015. Porous PES and PEI Hollow Fiber Membranes in a GasLiquid Contacting Process-A Comparative Study. J. Membr. Sci. 475: 57-64.

[16] G. Bakeri, D. Rana, A. F. Ismail, T. Matsuura, A. Ghaee. 2013. Performance of Surface-modified Poly(etherimide) Hollow-fiber Membranes in a Membrane GasLiquid Contacting Process with Response Surface Methodology. J. Appl. Polym. Sci. 128: 13131325.

[17] H. Zhu, X. Jie, L. Wang, G. Kang, D. Liu, Y. Cao. 2016. Effect of 
MIL-53 on Phase Inversion and Gas Separation Performance of Mixed Matrix Hollow Fiber Membranes. RSC Adv. 6: 6912469134.

[18] H. Dzinun, M. H. D. Othman, A. F. Ismail, M. H. Puteh, M. A. Rahman,
J. Jaafar. 2015. Morphological Study of Co-extruded Dual-layer Hollow Fiber Membranes Incorporated with Different $\mathrm{TiO}_{2}$ loadings. J. Membr. Sci. 479: 123131. 